STRUCTURAL BIOLOGY

ISSN 2059-7983

Received 14 November 2020

Accepted 23 February 2021

Edited by P. da Fonseca, University of Glasgow, United Kingdom

Keywords: single-particle cryo-EM; high throughput; high-resolution $3 \mathrm{D}$ reconstruction.

EMDB reference: apoferritin, EMD-12358

Supporting information: this article has supporting information at journals.iucr.org/d

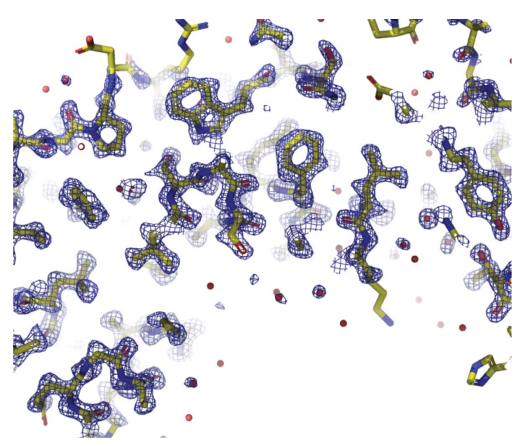

OPEN $\odot$ ACCESS

\section{Coma-corrected rapid single-particle cryo-EM data collection on the CRYO ARM 300}

\author{
Rouslan G. Efremov ${ }^{a, b *}$ and Annelore Stroobants ${ }^{a, b}$ \\ ${ }^{\mathbf{a}}$ Center for Structural Biology, Vlaams Instituut voor Biotechnologie, Pleinlaan 2, 1050 Brussels, Belgium, and ${ }^{\mathbf{b}}$ Structural \\ Biology Brussels, Vrije Universiteit Brussel, Pleinlaan 2, 1050 Brussels, Belgium. *Correspondence e-mail: \\ rouslan.efremov@vub.be
}

Single-particle cryogenic electron microscopy has recently become a major method for determining the structures of proteins and protein complexes. This has markedly increased the demand for throughput of high-resolution electron microscopes, which are required to produce high-resolution images at high rates. An increase in data-collection throughput can be achieved by using large beamimage shifts combined with off-axis coma correction, enabling the acquisition of multiple images from a large area of the EM grid without moving the microscope stage. Here, the optical properties of the JEOL CRYO ARM 300 electron microscope equipped with a K3 camera were characterized under offaxis illumination conditions. It is shown that efficient coma correction can be achieved for beam-image shifts with an amplitude of at least $10 \mu \mathrm{m}$, enabling a routine throughput for data collection of between 6000 and 9000 images per day. Use of the benchmark for the rapid data-collection procedure (with beam-image shifts of up to $7 \mu \mathrm{m}$ ) on apoferritin resulted in a reconstruction at a resolution of $1.7 \AA$. This demonstrates that the rapid automated acquisition of high-resolution micrographs is possible using a CRYO ARM 300.

\section{Introduction}

Density maps of proteins can be reconstructed at atomic resolution using single-particle cryogenic electron microscopy (cryo-EM; Nakane et al., 2020; Yip et al., 2020). Although intense development of the method continues, single-particle cryo-EM has already revolutionized structural biology over the course of the last six years. Today, obtaining 3D reconstructions of proteins and protein complexes at near-atomic resolution (better than $4 \AA$ ) has become routine. The rapid progress in single-particle cryo-EM has been enabled by the introduction and perfection of direct electron detectors (Ruskin et al., 2013) and by improvements in image-processing software (Scheres, 2012; Grant et al., 2018; Punjani et al., 2017). High-resolution imaging imposes stringent constraints on electron microscopes, as images with minimal subatomic drift and minimal aberrations are required to preserve information at atomic resolution $(\sim 1 \AA)$. The development of dedicated high-resolution, stable and fully automated electron microscopes, which are equipped with energy filters and produce highly coherent parallel illumination over large illumination areas, has also played a critical role in the success of the method.

The collection of a single-particle data set involves the acquisition of thousands of images from a vitrified protein solution on a cryo-EM grid, and this process is enabled by highly reliable automation of the data-collection process. Multiple software packages facilitating automated data collection, including, but not limited to, Leginon (Suloway et 
al., 2005), SerialEM (Mastronarde, 2005) and EPU (Thermo Fisher Scientific), have been developed. These software packages allow their users to visualize the EM grid at different magnifications. Initially, an atlas of the complete grid is recorded. This is used to select good grid squares before the positions for image recording are chosen. Automated data collection occurs next, executing a protocol with defined dataacquisition parameters.

Modern crystallographic beamlines on synchrotrons have a very high throughput: the collection of a diffraction data set takes only a few minutes (Svensson et al., 2015), whereas the collection of single-particle cryo-EM data sets often takes days. The lower single-particle cryo-EM data-collection throughput is because of the necessity of collecting thousands of images at spatially separated positions on a cryo-EM grid. This is achieved by displacing the sample stage with submicrometre accuracy, followed by a delay to stabilize stage drift (Suloway et al., 2005).

Recently, a new data-collection strategy has been introduced. It uses a beam-image shift with an amplitude ranging from few micrometres to tens of micrometres to collect singleparticle images from a large area of the EM grid without displacing the sample stage (Wu et al., 2019; Cheng et al., 2018). This approach significantly increases the data-collection throughput. The beam-image shift, however, induces coma and astigmatism (Wu et al., 2019; Cheng et al., 2018; Glaeser et al.,2011). Coma results in a phase error that grows at third and higher powers of spatial frequency (Glaeser et al., 2011); therefore, it primarily influences high-resolution shells (4 $\AA$ and higher) of the $3 \mathrm{D}$ reconstruction and becomes increasingly strong as one approaches atomic resolution (Zivanov et al., 2020). Beam-image shift-induced aberrations need to be optically corrected in order to maintain the high quality of the homogeneous single-particle data over the entire area used for data collection. The accuracy of the beam-image shift-induced coma correction, in combination with the absence of image shift-dependent higher-order aberrations (which cannot be corrected for), defines the success of this strategy for singleparticle data collection.

The JEOL CRYO ARM 300 is a new-generation $300 \mathrm{kV}$ electron cryogenic microscope released in 2018. It is equipped with a cold field emission gun (cFEG), an in-column $\Omega$ energy filter, a four-lens condenser and an autoloader for 12 samples. It has been shown that the CRYO ARM 300 enables 3D reconstruction to a resolution of $1.54 \AA$ for apoferritin (Kato et al., 2019). The performance of the CRYO ARM 300 has been characterized in multiuser environment settings (Fislage et al., 2020; Hamaguchi et al., 2019), but not for highthroughput data collection using coma-corrected beam-image shift.

Here, we describe procedures for rapid data collection using a coma-corrected beam-image shift on a CRYO ARM 300 using SerialEM. We characterized the optical properties of our CRYO ARM 300 microscope with applied beam-image shifts and assessed the efficiency of image shift-induced coma and astigmatism correction. Furthermore, we benchmarked this data-collection strategy with image shifts of up to $7 \mu \mathrm{m}$ on mouse heavy-chain apoferritin and achieved a reconstruction at a resolution of $1.7 \AA$. Altogether, our results demonstrate the suitability of a CRYO ARM 300 for rapid and highresolution single-particle data collection. They also indicate the limiting factors which need to be eliminated to improve both the throughput of data collection and the resolution of single-particle 3D reconstructions.

\section{Materials and methods}

\subsection{Purification of apoferritin and preparation of cryo-EM grids}

The plasmid with mouse heavy-chain apoferritin was a gift from Dr H. Yanagisawa (University of Tokyo). Apoferritin was expressed and purified following a procedure described previously (Fislage et al., 2020), with the only modification being that the reducing agent tris(2-carboxyethyl)phosphine (TCEP) was added at a concentration of $1 \mathrm{~m} M$ to the purification buffers to prevent protein aggregation during the preparation of the cryo-EM sample (Wu et al., 2020).

Cryo-EM grids were prepared by applying $3 \mu \mathrm{l}$ purified apoferritin at a concentration of approximately $3.6 \mathrm{mg} \mathrm{ml}^{-1}$ to 200-mesh holey Quantifoil R 1.2/1.3 carbon grids that had been glow-discharged for $1 \mathrm{~min}$ in an ELMO glow-discharge system (Cordouan). The grids were blotted with Whatman Grade 1 paper for $5 \mathrm{~s}$ with offset 0 at $25^{\circ} \mathrm{C}$ and $98 \%$ humidity, after which they were immediately plunged into liquid ethane at $-170^{\circ} \mathrm{C}$ using a Cryoplunge 3 (Gatan).

\subsection{Measurements of beam-image shift-induced coma and astigmatism}

Measurements of coma and astigmatism, as well as the acquisition of an apoferritin data set, were performed on a CRYO ARM 300 with spot size 6 , using alpha angle 1 with a condenser aperture of $150 \mu \mathrm{m}$ and the objective aperture retracted. The energy-filter slit was centered on the zero-loss peak, and the slit width was set to $20 \mathrm{eV}$. Images were recorded on a K3 detector (Gatan) operating in correlated double-sampling (CDS) mode, with an exposure of $3.37 \mathrm{~s}$ partitioned into 59 frames. A dose of approximately $1 \mathrm{e}^{-} \AA^{-2}$ was exposed per frame, at a nominal magnification of 60000 and with a corresponding magnified pixel size of $0.75 \AA$.

Beam-image shift-induced coma was measured using a grating grid (Electron Microscopy Sciences) with latex spheres. Beam-image shifts with a radius of between 2 and $10 \mu \mathrm{m}$, in $2 \mu \mathrm{m}$ steps, were applied along 16 equi-angularly spaced directions (angular step 22.5 ) and the beam tilt was measured using the SerialEM3.8.0 command FixComaByCTF. After coma correction, an image was recorded and the astigmatism was measured by fitting the power spectrum using CTFFIND4 (Rohou \& Grigorieff, 2015) after frame alignment in MotionCor2 (Zheng et al., 2017). The astigmatism angle was defined as per the study conducted by Mindell \& Grigorieff (2003) and the amplitude was calculated as the difference between the maximum and minimum values of defocus. The measurements were performed automatically 
using a home-written script both with and without applying coma and astigmatism correction. For the measurements, the grid $z$-height was offset from the standard focus by a distance of between 1 and $2 \mu \mathrm{m}$, and the current in the objective lens was close to the standard focus, within an equivalent defocus of $1 \mu \mathrm{m}$.

\subsection{Measurements of residual beam-image shift}

Residual drift, arising from the potential relaxation of the deflectors after setting the beam-image shift with the corresponding adjustment of the beam tilt and astigmatism, was measured in SerialEM3.8.0. For these measurements, additional delays incorporated in SerialEM were zeroed by setting the image-shift delay factor and the property AstigBTBacklashDelay to 0 .

A script implementing beam-image shift from the neutral position to a position with a given amplitude of image shift, and at equally spaced angles, was applied. This was followed by immediate image recording of the grating grid on the K3 detector operating in CDS mode, with a total exposure of $4.98 \mathrm{~s}$ partitioned over 87 frames and a flux of approximately $1 \mathrm{e}^{-} \AA^{-2}$ per frame. The time for each cycle of script execution was verified to ensure the absence of any additional delays, and it was found to be equal to the sum of the time required for both beam-image tilt-astigmatism application and image recording to within 0.4-0.6 s. The recorded frames were aligned in MotionCor2, and the resulting trajectory was used to analyze the residual image shift.

\subsection{Pivot-point alignment}

An accurate alignment of compensation for beam shift is critical for minimizing beam-shift-induced coma. To align the pivot points, the grid was brought to standard focus height and the beam diameter was carefully adjusted by minimizing the beam-diameter variation during objective lens wobbling, ensuring that the beam was parallel. The objective lens was defocused to between 40 and $100 \mu \mathrm{m}$. Next, the condenser aperture was removed and the beam shift was wobbled using SerialEM, with the largest amplitude maintaining the fluorescent screen illuminated at the extreme beam positions. The beam-shift compensator was adjusted to minimize the movement of a feature on the grid along the wobbling direction.

Following the adjustment of pivot points, refinement of the beam shift against image shift at large image-shift amplitudes (up to $10 \mu \mathrm{m}$ ) was performed in SerialEM to ensure precise beam centering at large beam-image shifts using the SerialEM Refine Beam Shift Cal command.

\subsection{Single-particle data collection and image processing}

A single-particle cryo-EM data set for apoferritin was collected using the CRYO ARM 300 electron microscope at a nominal magnification of 60000 . The microscope illumination conditions were set to spot size 6 and alpha 1. A condenser aperture of $150 \mu \mathrm{m}$ was used to produce an illumination area of approximately $1.5 \mu \mathrm{m}$ in diameter, and the objective aperture was retracted. The energy-filter slit was centered on the zero-loss peak, with the slit width set to $20 \mathrm{eV}$. Images were recorded on a $\mathrm{K} 3$ detector (Gatan) operating in CDS mode, with an exposure time of $3.37 \mathrm{~s}$ partitioned onto 59 frames. The corresponding electron dose was $1 \mathrm{e}^{-} \AA^{-2}$ per frame, the corresponding flux on the detector was $10 \mathrm{e}^{-}$per pixel per second and the calibrated magnified pixel size was $0.753 \AA$.

Before data collection, calibration of coma and astigmatism matrices versus image shift was performed in SerialEM3.8.0 using a grating grid. Coma-free alignment was performed before launching the data collection. Data were collected using SerialEM3.8.0 from a pattern of $5 \times 5$ holes on a Quantifoil R 1.2/1.3 grid, recording one image in the center of each hole. The holes were sampled along a serpentine-shaped path starting from a corner of the pattern. Focusing was performed once per stage position on the carbon area next to the central hole. Focusing within a limit of $\pm 5 \mu \mathrm{m}$ from the standard focus was performed by adjusting the objective lens current. For larger defoci, the $z$-height of the stage was adjusted before the objective lens current was used to focus. A data set of 3125 images was collected from 125 stage positions. After frame alignment in MotionCor 2 and determination of CTF parameters in CTFFIND4 (Rohou \& Grigorieff, 2015), the images were assessed with the help of BXEMDALYSER (a home-written program that allows the user-friendly selection of micrographs based on their visual appearances, parameters of frame alignment and CTF fits; unpublished work). Images with thick or crystalline ice and those containing multiple layers of apoferritin particles were discarded.

From the remaining 2639 micrographs, a total of 1514772 particles picked in crYOLO1.5.6 (Wagner et al., 2019) were extracted in a box of 140 pixels binned twice. The particles were subjected to a 2D classification into 150 classes performed with the 'ignore until first CTF peak' option selected in RELION3.1 (Zivanov et al., 2020). Further processing was performed in RELION3.1. After two rounds of 2D classification, 1326632 particles were selected for further processing. Unmasked initial 3D auto-refinement, performed on particles extracted into a box size of 280 pixels and against the initial model filtered to $40 \AA$, converged to a resolution of 2.6 A. Refinement of beam tilt indicated an $X / Y$ tilt of $-0.25 /$ $-0.65 \mathrm{mrad}$. After tilt correction and per-particle defocus refinement, the resolution of $3 \mathrm{D}$ reconstruction improved to $2.1 \AA$ A.

Following Bayesian polishing and another round of CTF refinement, including trefoil and fourth-order aberration, anisotropic magnification and retention of particles with defocus below $1.2 \mu \mathrm{m}$ to avoid CTF aliasing (Penczek et al., 2014), a total of 702667 particles were retained and the resolution further improved to $1.79 \AA$ A. Next, individual optics groups were created for data collected from each stage position and the beam tilt was refined for each optics group. This further improved the resolution to $1.71 \AA$. The Rosenthal-Henderson $B$ factor was calculated using the bfactor_plot.py script (Zivanov et al., 2018). Real-space refinement of the apoferritin model (PDB entry 3wnw; R. Zarivach \& L. Lewin, unpublished work) against the final 
map was performed in Phenix1.14 (Afonine et al., 2013), and the map-model FSC was calculated using the phenix.mtriage routine (Afonine et al., 2018).

To evaluate the quality of single-particle data recorded at individual positions in the $5 \times 5$ pattern, the polished particles were split into optics groups containing images with identical beam-image shifts. Beam tilt and higher-order aberrations were refined for each optics group. Next, 3D auto-refinement with local angular sampling, starting from $1.8^{\circ}$, was calculated for each optics group separately. This was followed by calculation of the Rosenthal-Henderson $B$ factor for each subset.

\section{Results}

\subsection{How fast can single-particle data be collected?}

We first asked the question: how fast can single-particle data be collected? In the process of single-particle data collection, multiple operations are executed on the microscope at different frequencies. To optimize throughput, it is helpful to have a model that can be used to evaluate the dependence of the throughput on the duration of the individual steps.

Table 1 summarizes the individual steps involved in the data-collection process, as well as the characteristic times per step measured for the procedures as established using our microscope. The throughput, $N$, is defined as the number of micrographs collected per $24 \mathrm{~h}$ and can be estimated using

$$
N=\frac{24-t_{\mathrm{LN}}}{\left(t_{\text {cycle }} / n\right)+t_{\text {img }}},
$$

where $n$ is the number of micrographs recorded per stage position, $t_{\text {cycle }}=t_{\text {stage }}+t_{\text {focus }}+t_{\text {beam }}+t_{\text {ice }}$ is the cumulative time of operations performed only once per stage position, and $t_{\text {img }}=t_{\mathrm{BS}}+t_{\mathrm{stab}}+t_{\mathrm{rec}}+t_{\text {SerialEM }}$ is the cumulative time for collecting one micrograph (units of time should be measured in hours for this equation). The corresponding values of $t_{\text {cycle }}$ and $t_{\text {img }}$ for the data-collection routine set up on our microscope are 66 and 7.5-9.8 s, respectively. The dependence of the throughput on the number of micrographs recorded per stage position, for the combination of parameters discussed below, is shown in Fig. 1. The throughput increases rapidly as the number of images acquired per stage position increases, and for values of $n$ such that $n \gg t_{\text {cycle }} / t_{\text {img }}$, the throughput is defined by $t_{\mathrm{img}}$ only. The rate of increase in throughput depends on the ratio $t_{\text {cycle }} / t_{\text {img }}$, and it reaches half-maximum when $n$ is equal to $t_{\text {cycle }} / t_{\text {img }}$ (between 7 and 8 for the timings listed in Table 1).

When a few tens, or more, of images are recorded per stage position, $t_{\text {img }}$ becomes the main determinant of data-collection throughput (equation 1 and Fig. 1). Therefore, we looked closely at the duration of the individual steps contributing to $t_{\text {img. }}$. The time required for image recording $\left(t_{\text {rec }}\right)$, the main contributor to $t_{\text {img }}$, consists of the actual exposure time plus the dead time of the camera. For the flux recommended for the $\mathrm{K} 3$ camera (between 8 and $40 \mathrm{e}^{-}$per pixel per second), a pixel size of $0.75 \AA$ (corresponding to a magnification of 60000 used for routine data collection on our microscope) and a
Table 1

Timing of individual operations during single-particle data collection.

\begin{tabular}{llll}
\hline Operation & Duration & Frequency & Symbol \\
\hline Liquid-nitrogen dewar refilling & $20 \mathrm{~min}$ & Every $13 \mathrm{~h}$ & $t_{\mathrm{LN}}{ }^{\dagger}$ \\
Stage positioning & $28 \mathrm{~s}$ & Per cycle & $t_{\text {stage }}$ \\
Focusing & $26 \mathrm{~s}$ & Per cycle & $t_{\text {focus }}$ \\
Beam centering & $6 \mathrm{~s}$ & Per cycle & $t_{\text {beam }}$ \\
Beam shift/tilt/stigmator & $4 \mathrm{~s}$ & Per image & $t_{\mathrm{BS}}$ \\
Stabilization delay & $0 \mathrm{~s}$ & Per image & $t_{\text {stab }}$ \\
Image exposure and recording & $3.3-5.6 \mathrm{~s}$ & Per image & $t_{\text {rec }} \ddagger$ \\
SerialEM cycle overhead & $0.15 \mathrm{~s}$ & Per image & $t_{\text {SerialEM }}$ \\
Ice-thickness measurement & $5.5 \mathrm{~s}$ & Per cycle & $t_{\text {ice }}$ \\
\hline
\end{tabular}

$\dagger t_{\mathrm{LN}}$ in (1) signifies the total average time spent in refilling liquid-nitrogen dewars and stage stabilization every $24 \mathrm{~h}$. $\ddagger$ A range of times is shown for the shortest and longest exposure times used on our microscope.

cumulative electron dose of $\sim 60 \mathrm{e}^{-} \AA^{-2}$, the exposure times are between 2.15 and $3.38 \mathrm{~s}$. An additional overhead of approximately $2 \mathrm{~s}$, which does not depend on the exposure settings, is associated with the recording of an image (Supplementary Table S1). Reducing the total exposure to $30 \mathrm{e}^{-} \AA^{-2}$, which is common for single-particle data-collection procedures, can further reduce the image-recording time to $3.3 \mathrm{~s}$.

After eliminating all of the delays encoded in SerialEM during the process of setting the beam-image shift and adjusting the beam tilt and astigmatism, $t_{\mathrm{BS}}$ was measured to be $4 \mathrm{~s}$. This time is surprisingly long, and considerably impacts the saturation throughput, creating an unexpected bottleneck in the data-collection throughput. We experimentally verified whether the stabilization delay $\left(t_{\text {stab }}\right)$, the time after application of the beam-image shift and before acquisition of the EM

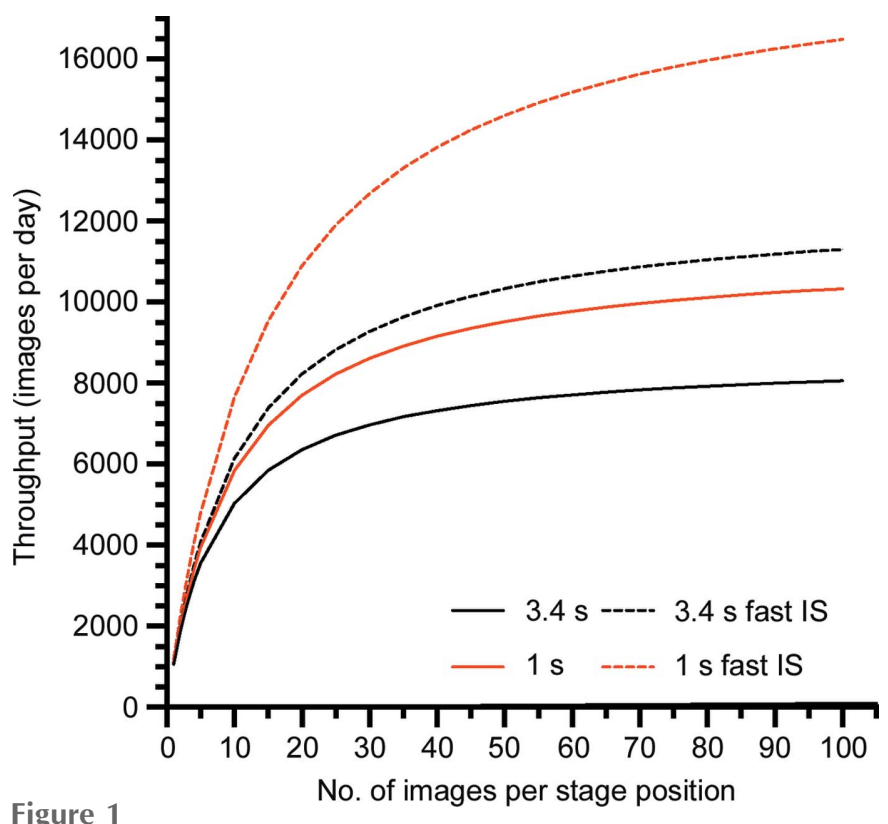

Figure 1

Daily throughput of micrograph acquisition as a function of number of images recorded per stage position. Curves are shown for exposure times of 3.4 and $1 \mathrm{~s}$ for the currently set up data-acquisition procedure (solid lines). The corresponding saturation throughputs are 8600 and 11300 images per day, respectively. Dashed lines show hypothetical throughputs, assuming that $t_{\mathrm{BS}}$ is reduced from 4 to $1 \mathrm{~s}$. The corresponding saturation throughputs are 12500 and 18900 images per day, respectively. 
image, is needed to ensure that potential relaxation of deflectors does not cause residual image drift. To do this, a movie was recorded on a grating immediately after shifting the beam, adjusting the beam tilt and astigmatism for various directions and the amplitudes of the beam-image shift. If a significant residual relaxation of image shift occurred, an apparent residual drift should be visible on the trajectories of the aligned frames. Supplementary Fig. S1 shows that after an image shift of $10 \mu \mathrm{m}$, a linear drift is observed over a distance of between 0.2 and $1.5 \AA$. Because of its variable amplitude and the absence of correlation between the direction of image

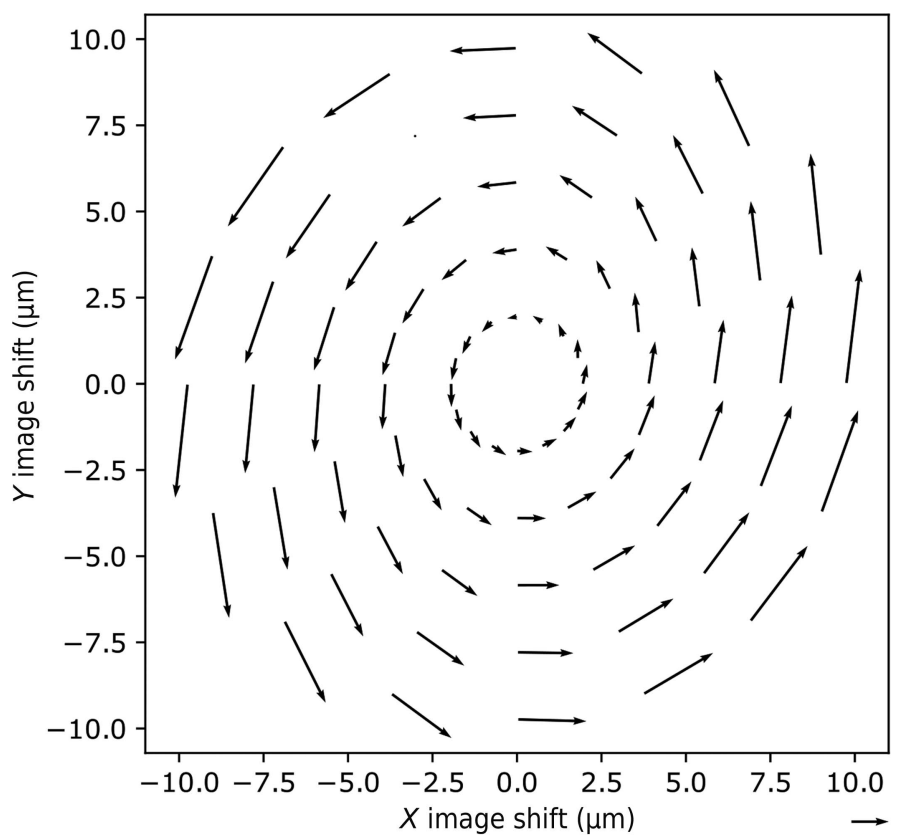

(a)

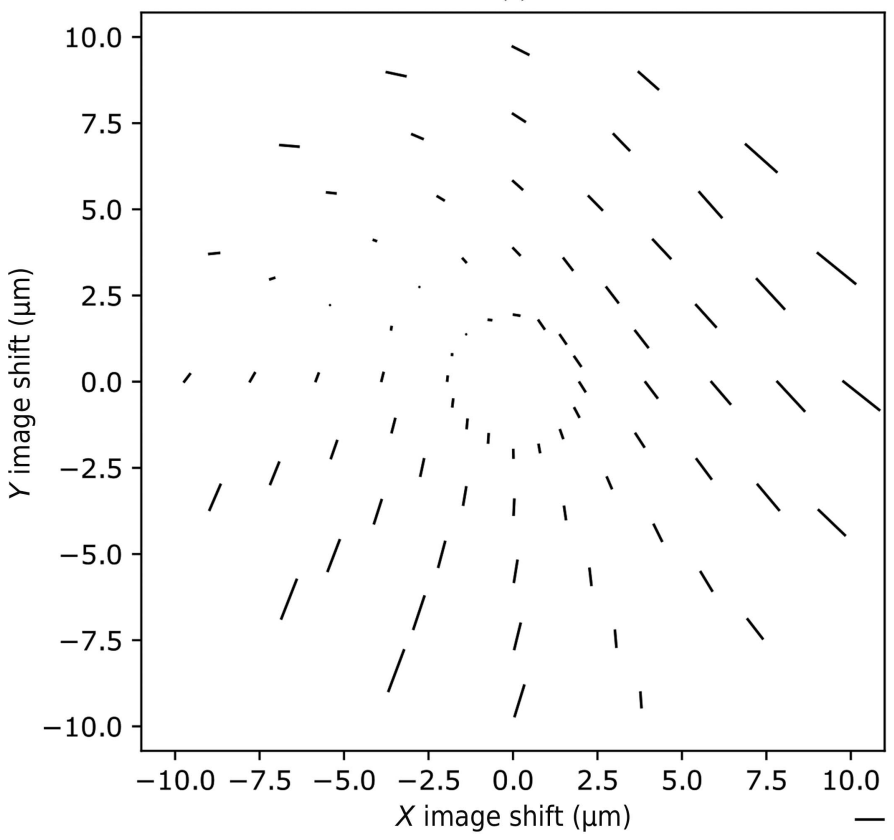

(c) shift and the direction of apparent drift, it is most likely caused by a charging effect rather than by relaxation of the deflectors. Moreover, owing to the high rate of fractionation, the associated shifts per frame were always below $0.4 \AA$, which does not influence the image quality at resolutions lower than $1.2 \AA$ (Fislage et al., 2020). Thus, we can conclude that deflectorstabilization delay $\left(t_{\text {stab }}\right)$ is not required during data collection.

In conclusion, given the parameters established for datacollection procedures on our CRYO ARM 300, throughput limits of between 8600 and 11300 images per day can be expected. In practice, for a limited number of images recorded

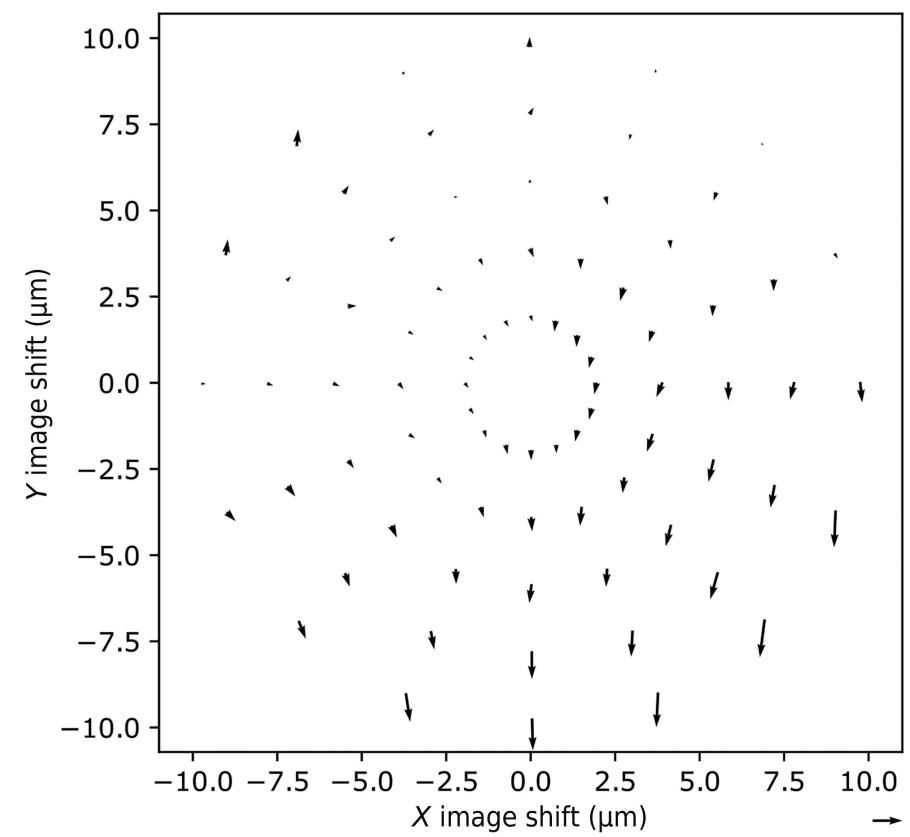

(b)

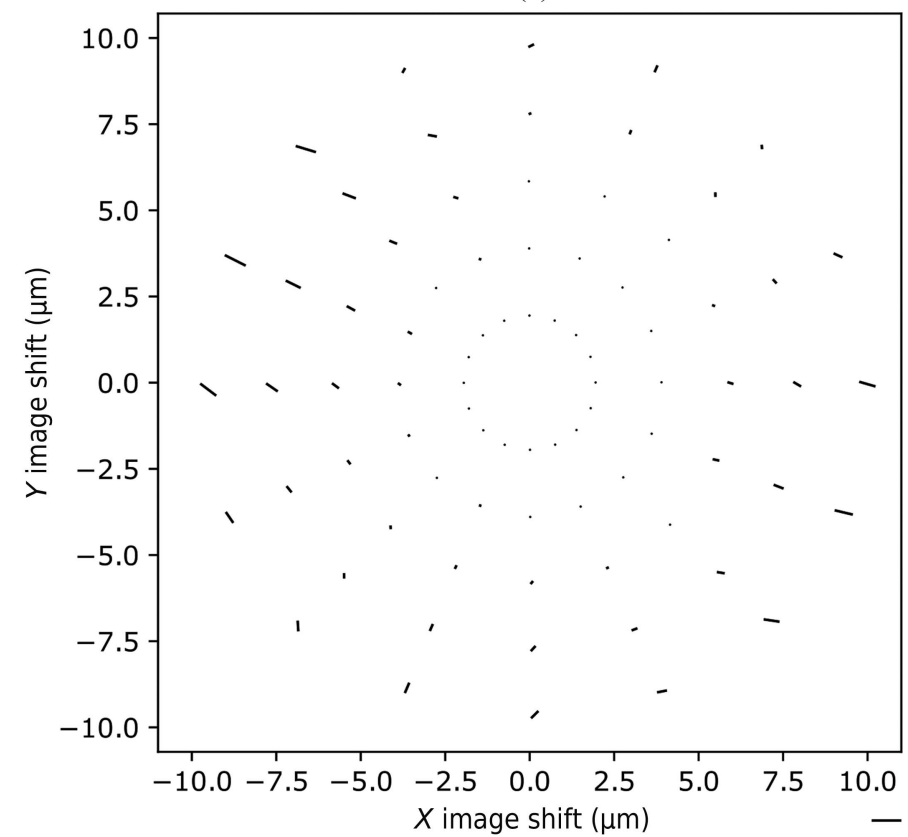

(d)

Figure 2

Vector plots of beam-image shift-induced coma and astigmatism. Vectors indicate the amplitude and direction of the beam-image shift-induced beam tilt $(a)$ before and $(b)$ after correction. The scale bar for $(a)$ is $1 \mathrm{mrad}$ and that for $(b)$ is $0.2 \mathrm{mrad}$. Lines show the amplitude and direction of astigmatism, as defined by Mindell \& Grigorieff (2003), $(c)$ before and $(d)$ after correction. The scale bars in both panels are $2000 \AA$. 
per stage position, throughputs of between 6000 and 9000 images per day can be reached. It is important to note, however, that reducing $t_{\mathrm{BS}}$ from 4 to $1 \mathrm{~s}$ would further accelerate the collection rate to the range of between 9000 and 17000 images per day (Fig. 1).

\subsection{Beam-image shift-induced coma and its correction}

Moving the beam away from the optical axis induces coma and astigmatism, the magnitudes of which depend on the microscope alignment and the properties of the objective lens (Glaeser et al., 2011; Cheng et al., 2018). Both aberrations can be efficiently corrected for: changing the beam tilt corrects for coma and adjusting the objective lens stigmators can compensate for astigmatism (Wu et al., 2019).

The range of accessible beam shifts on the CRYO ARM 300 has limits that depend on the illumination angle alpha. Thus, at alpha 1 , which is typically used for data collection with a K3 detector, the beam-shift amplitude is limited to approximately

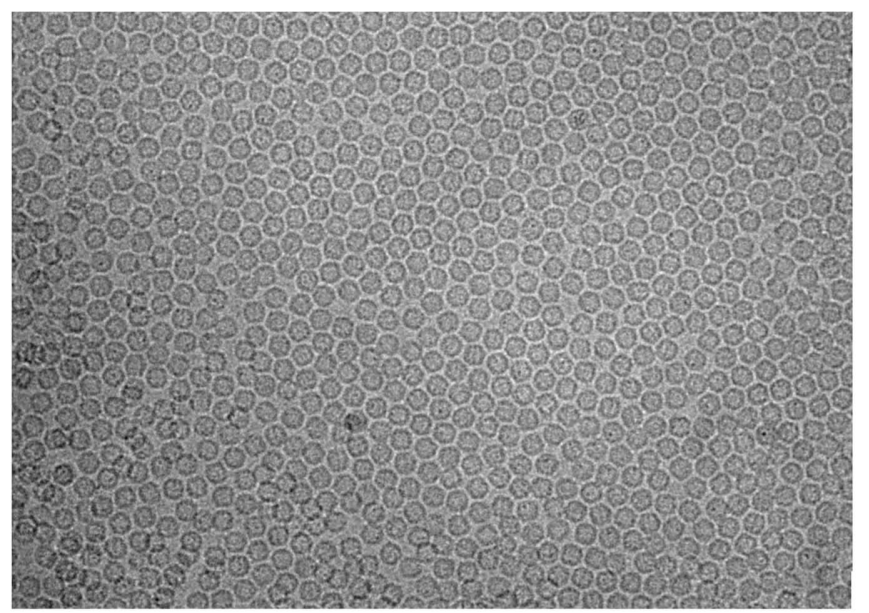

(a)

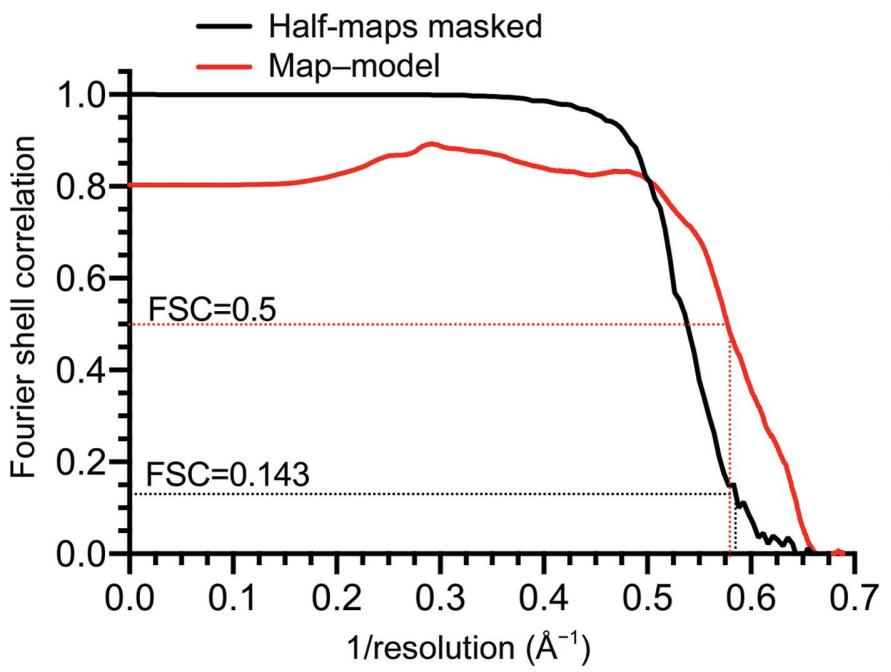

(c)
$15 \mu \mathrm{m}$. At alpha 2, however, the limit extends to approximately $40 \mu \mathrm{m}$. Therefore, alpha 2 may need to be used for beam-image shifts with amplitudes above $15 \mu \mathrm{m}$.

Inaccuracy in the alignment of pivot points contributes to beam-induced coma. We have noticed that the standard pivotpoint alignment procedure, which utilizes the diffraction mode, was not accurate enough and had to be improved. Following the suggestion of Dr Sohei Motoki (JEOL), we modified the alignment procedure to adjust image-shift compensation in imaging mode. This modification is described in more detail in Section 2. To evaluate the optical properties of the microscope, we measured the beam-image shift-induced coma and astigmatism for a range of image shifts up to $10 \mu \mathrm{m}$ (Fig. 2a).

The beam-image shift-induced coma was nearly perpendicular to the direction of the image shift, as existing theory had led us to expect (Glaeser et al., 2011), and had an elliptical dependence on the amplitude of the image-shift direction (Supplementary Fig. S2a). Coma amplitude increases linearly

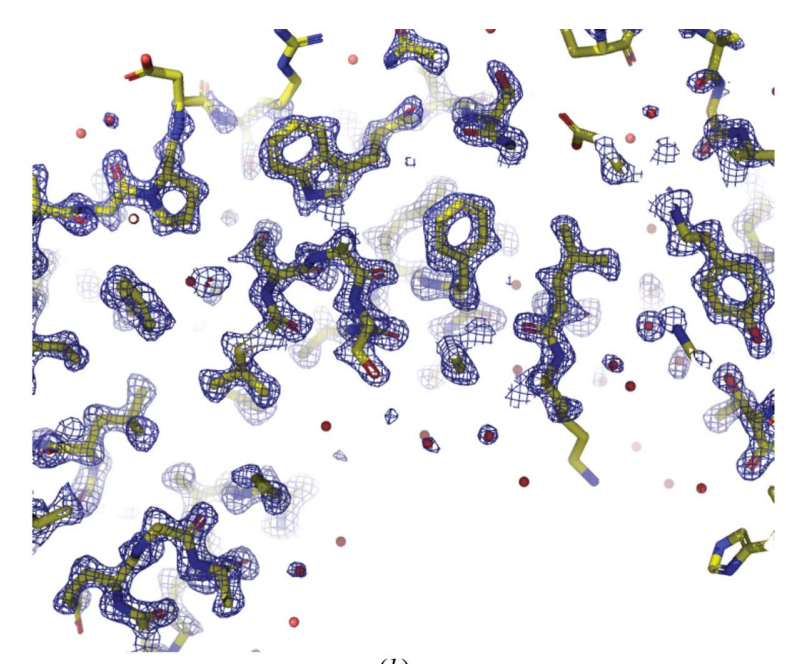

(b)

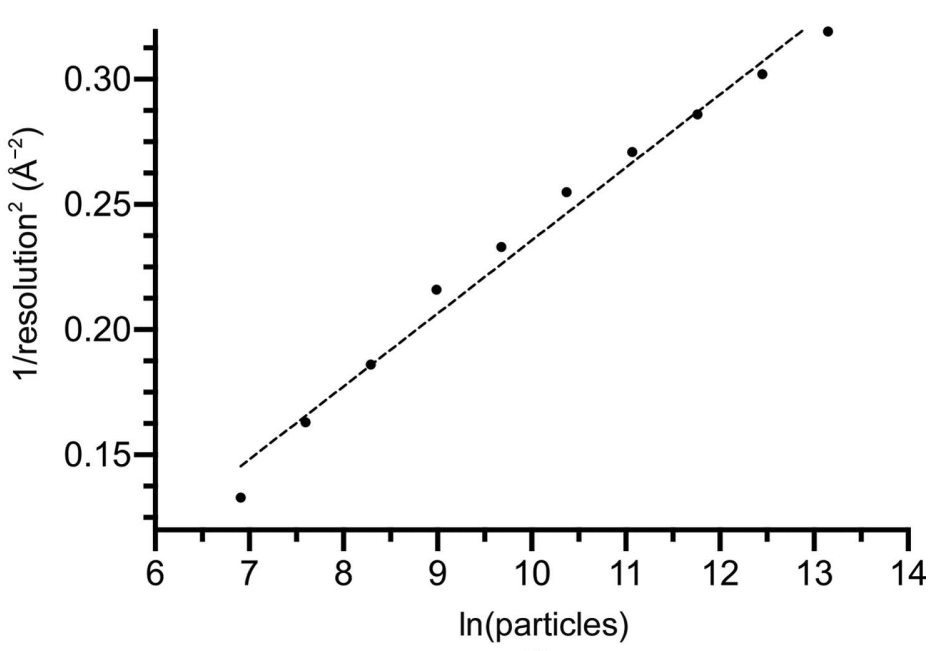

(d)

Figure 3

Reconstruction of apoferritin from a data set collected from a $5 \times 5$ pattern on a Quantifoil R 1.2/1.3 grid. (a) Example micrograph of apoferritin. (b) Density map at a resolution of $1.7 \AA$. (c) Fourier shell correlation curve for the masked reconstruction and between the refined model and the map. $(d)$ Rosenthal-Henderson $B$-factor plot fitted with a $B$ factor of $68 \AA^{2}$. 
as the amplitude of the image shift increases, with slopes of

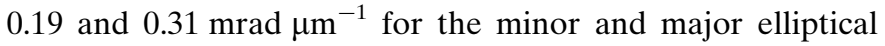
axis directions, respectively (Supplementary Fig. S2c).

The coma remaining after applying beam-tilt correction (using a linear matrix dependent on image shifts), as implemented in SerialEM3.8.0, is shown in Fig. 2(b) and Supplementary Fig. S2(c). The fivefold difference in the length of the scale bar between Figs. 2(a) and 2(b) should be noted. For most image-shift positions, beam-tilt correction reduces the coma to below $0.1 \mathrm{mrad}$. However, a certain level of residual coma remains for some directions, revealing an asymmetry in the beam-image shift-induced coma. The center of the residual coma pattern is shifted towards the top left corner of the sampled beam-image shift space, and the residual coma reaches up to $0.25 \mathrm{mrad}$ at the largest beam-image shift. However, these values of residual coma should still allow single-particle reconstruction to a resolution above $2.4 \AA$ (Glaeser et al., 2011).

The pattern of beam-image shift-induced astigmatism is significantly more asymmetric than that of coma (Fig. $3 c$, Supplementary Fig. S3a) The amplitude of astigmatism reaches up to $3500 \AA$ for an image shift of $10 \mu \mathrm{m}$. The center of the astigmatism pattern appears to be located in roughly the same position as the center of the residual coma pattern (Fig. 3b), suggesting that the asymmetric behaviors of both coma and astigmatism may share an origin. In spite of its asymmetric character, astigmatism can be efficiently reduced by adjusting the objective lens stigmators using a simple linear transformation of image-shift values, as was implemented in SerialEM3.8.0 (Fig. 3d, Supplementary Fig. S3b). Upon correction, the astigmatism is reduced to below $300 \AA$ for image shifts up to $4 \mu \mathrm{m}$ and to below $1600 \AA$ for a beam-image shift with an amplitude of $10 \mu \mathrm{m}$ (Supplementary Fig. S3b). The magnitude of residual astigmatism is significantly lower than the amplitude of defocus ( $0.5 \mu \mathrm{m}$ and higher); therefore, if determined accurately by defocus-fitting programs, the residual astigmatism should not interfere with downstream image processing and CTF correction. More accurate astigmatism correction can be achieved by fitting beam-image shiftinduced astigmatism with a more sophisticated empirical function, as, for example, realized by Wu et al. (2019).

\subsection{Benchmark with apoferritin}

To benchmark rapid data collection with significant image shifts, we collected a data set for mouse heavy-chain apoferritin. The density of apoferritin particles on the cryo-EM grid improved compared with our previously reported benchmark (Fislage et al., 2020), resulting in a tightly packed layer of particles (Fig. 3a). This was achieved by adding a reducing agent to the purification buffers, similar to the work of Wu et al. (2020). Further details can be found in Section 2. It is likely that under oxidizing conditions a surface-exposed cysteine (Cys102) cross-links apoferritin molecules with cysteine bridges as the protein concentrates at the air-water interface during the preparation of the cryo-EM sample
Table 2

Data-collection parameters and statistics for the apoferritin data set.

\begin{tabular}{ll}
\hline Data collection & CRYO ARM 300 \\
Electron microscope & K3, CDS mode \\
Electron detector & 300 \\
Voltage $(\mathrm{kV})$ & 60000 \\
Nominal magnification & 3.37 \\
Exposure time $(\mathrm{s})$ & $0.3-1.5$ \\
Defocus range $(\mu \mathrm{m})$ & 25 \\
No. of exposures per stage position & 59 \\
No. of frames & 0.753 \\
Pixel size $(\AA)$ & 60 \\
Electron dose $\left(\mathrm{e}^{-} \AA^{-2}\right)$ & 3125 \\
No. of collected images & \\
3D reconstruction & 702667 \\
Final particles & $O$ \\
Applied symmetry & 1.71 \\
Resolution $(\AA)$ & -51 \\
Sharpening $B$ factor $\left(\AA^{2}\right)$ & 68 \\
Rosenthal-Henderson $B$ factor $\left(\AA^{2}\right)$ & \\
\hline
\end{tabular}

(Armstrong et al., 2019). This causes protein aggregation and a reduction of particle density in the holes of the EM grid.

We estimated that the objective aperture limits the resolution to the range between 1.5 and $2.0 \AA$. Therefore, the objective aperture was retracted for this benchmark. 25 images were recorded per stage position from a $5 \times 5$ array of holes on a Quantifoil R 1.2/1.3 grid. One image was recorded in the center of each hole of the array. With these settings, the largest image shifts were $7 \mu \mathrm{m}$ at the corners of the square pattern. The data set was collected with the beam size expanded to $1.5 \mu \mathrm{m}$ to cover the carbon area around the hole, which aimed to reduce the charging effect that we regularly observed with apoferritin samples. This is different from regular conditions, in which a beam with a diameter of $1 \mu \mathrm{m}$ is used, collecting three images per $1.2 \mu \mathrm{m}$ hole and five per $2 \mu \mathrm{m}$ hole.

A data set of 3125 images acquired from 125 stage positions was collected in $12 \mathrm{~h}$ (the refilling of liquid-nitrogen dewars occurred during this time). This corresponds to a dataacquisition rate of 6250 images per day, which is within a $10 \%$ margin of the expected rate of 6717 (Fig. 1). This datacollection rate is 2.6 times higher than the previous fastest data-collection rate that we have reported, which was achieved with a K2 camera and was collected by recording five images per stage position (Fislage et al., 2020).

A $3 \mathrm{D}$ reconstruction of apoferritin was calculated to a resolution of $1.71 \AA$ from 702667 particles imaged with a defocus below $1.2 \mu \mathrm{m}$ (Table 2, Fig. $3 b$ ). A $B$ factor of $68 \AA^{2}$ was also determined from the $B$-factor plot (Rosenthal \& Henderson, 2003; Fig. 3d). The 3D map displayed the features expected at this resolution (Fig. 3b), including the holes in the imidazole rings of histidines and in the five-membered pyrrole rings of tryptophan side chains. Many $\mathrm{C}$ atoms displayed separated density blobs at higher contour levels. Refinement of optical aberrations in accordance with the work of Zivanov et al. (2020) revealed a surprisingly high average beam tilt of $0.9 \mathrm{mrad}$ in the data set, which was present due to the instability of the beam tilt at the beginning of data collection. 
Table 3

Properties of apoferritin data acquired from individual positions of the $5 \times 5$ pattern averaged for positions with an identical amplitude of the beamimage shift.

\begin{tabular}{|c|c|c|c|c|c|c|c|}
\hline $\begin{array}{l}\text { Shift radius } \\
(\mu \mathrm{m})\end{array}$ & $\begin{array}{l}\text { No. of } \\
\text { images }\end{array}$ & $\begin{array}{l}\text { No. of particles } \\
\times 10^{3}\end{array}$ & $\begin{array}{l}\text { Astigmatism amplitude } \\
(\AA) \times 100\end{array}$ & $\begin{array}{l}\text { FSC resolution } \\
(\AA)\end{array}$ & $\begin{array}{l}\text { Sharpening } B \\
\text { factor }\left(\AA^{2}\right)\end{array}$ & $\begin{array}{l}\text { Rosenthal-Henderson } \\
B \text { factor }\left(\AA^{2}\right)\end{array}$ & $\begin{array}{l}\text { No. of averaged } \\
\text { positions }\end{array}$ \\
\hline 0 & 112 & 57.5 & 1.85 & 1.97 & 50 & 59 & 1 \\
\hline 2.5 & $112 \pm 5$ & $57 \pm 3$ & $2.1 \pm 0.5$ & $2.00 \pm 0.01$ & $52 \pm 2$ & $59 \pm 2$ & 4 \\
\hline 3.5 & $112 \pm 6$ & $57 \pm 4$ & $2.7 \pm 0.7$ & $1.99 \pm 0.02$ & $51 \pm 1$ & $61 \pm 4$ & 4 \\
\hline 5.0 & $104 \pm 8$ & $53 \pm 5$ & $3.7 \pm 1.2$ & $1.99 \pm 0.01$ & $51 \pm 1$ & $63 \pm 3$ & 4 \\
\hline 5.5 & $103 \pm 9$ & $52 \pm 5$ & $4.3 \pm 1.6$ & $2.02 \pm 0.03$ & $51 \pm 1$ & $62 \pm 3$ & 8 \\
\hline 7.0 & $98 \pm 15$ & $48 \pm 8$ & $6.3 \pm 2.0$ & $2.01 \pm 0.04$ & $51 \pm 2$ & $61 \pm 3$ & 4 \\
\hline
\end{tabular}

This beam-tilt instability on our microscope is a recurrent problem which requires further examination.

To obtain the reconstruction, processing with a single optics group was performed; that is, neither beam tilt nor aberration correction were refined separately for images recorded with a shifted beam. Before the last reconstruction, individual optics groups were created for groups of images recorded from the same stage position. The beam tilt for each group was refined independently to account for instabilities in beam tilt. The overall quality of these data is comparable to the reconstruction obtained from data recorded close to the optical axis and at higher magnification on a K2 detector (Fislage et al., 2020), indicating that larger image shifts do not cause the quality of the single-particle data to deteriorate.

To further understand whether the quality of the collected single-particle data was affected by beam-image shift, the optical properties for particles extracted from images recorded in individual positions in the $5 \times 5$ pattern were refined as independent optics groups. Thereafter, reconstructions were refined independently for the subsets of particles acquired at corresponding positions in the beam-image shift pattern (Table 3 and Supplementary Table S2). The number of extracted particles per position varied between 36000 and 60000 , and the reconstructions reached very similar resolutions of between 1.97 and $2.07 \AA$, where the difference in resolution correlates with the number of particles in the data sets. Their sharpening and Rosenthal-Henderson $B$ factors had only a narrow spread in the ranges $49-56$ and $55-61 \AA^{2}$, respectively (Table 3, Supplementary Table S2). This suggests that the quality of the data recorded with large beam-image shifts is homogeneous within a $\pm 7 \mu \mathrm{m}$ range and is directionindependent (Table 3, Supplementary Fig. S4). It is notable that the beam tilts refined for individual positions deviate from the average beam tilt by a maximum of $0.08 \mathrm{mrad}$. This indicates that the coma correction is accurate and stable over the period of data collection and is not a resolution-limiting factor at least to a resolution of $1.7 \AA$. No significant variations were observed for the higher-order aberrations, including trefoil and fourth-order aberrations, determined in relation to the data recorded at different positions of the $5 \times 5$ pattern (Supplementary Table S2). Indeed, refinement of the complete data set with 25 optical groups, and independent tilt refined for each group, improved the resolution by only $0.04 \AA$.

The average residual astigmatism in individual positions of the $5 \times 5$ pattern ranges from 140 to $870 \AA$ (Table 3 and
Supplementary Table S2; as expected from the measurements shown in Fig. $2 d$ and Supplementary Fig. S3b) and does not influence the quality of the single-particle reconstruction.

Cumulatively, this analysis indicates that coma can reliably be corrected on the CRYO ARM 300 for image-shift amplitudes of at least $7 \mu \mathrm{m}$ and that the information in the images is preserved at least to a resolution of $1.7 \AA$.

\section{Discussion}

Here, we characterized the performance of the CRYO ARM 300 electron microscope for single-particle data collection using coma- and astigmatism-corrected image shifts with amplitudes of up to $7 \mu \mathrm{m}$. This data-collection strategy allows the recording of multiple images from a single stage position, thus significantly increasing the throughput. We show that using the data-collection procedures established on our CRYO ARM 300, single-particle data can be routinely collected with throughputs of between 6000 and 9000 images per day. This is a more than 2.6-fold increase in data-collection throughput compared with previously reported data-collection rates (Fislage et al., 2020).

This acceleration is due to a combination of factors. Although an increased number of images per stage position was the main focus of throughput improvement, a faster detector (K3 versus $\mathrm{K} 2$ ) that roughly quartered the exposure and image-recording time also contributed. A reduction of the time needed for stage alignment and the elimination of other unnecessary delays also played important roles. Analysis of the throughput clearly showed that one of the bottlenecks is associated with excessive time expenditure, needing an additional $4 \mathrm{~s}$ to set the beam-image shift, tilt and astigmatism of the electron beam. While this manuscript was under revision, JEOL updated the TEM Center from version 4.2.2 to 4.2.3, and the dead time associated with beam manipulation was reduced to approximately $0.6 \mathrm{~s}$. As a result, data-collection throughput further increased to around 8000 images per day when using a data-collection strategy that acquired 20 images per stage position. This rate could be predicted using our model (Fig. 1, red curves), and it can now easily exceed 10000 images per day. Our measurement shows that with a faster response time, the deflectors do not display any residual relaxation of image shift (Supplementary Fig. S5). Therefore, faster data collection should not compromise the quality of recorded micrographs. 
We have characterized the optical properties of the microscope for beam-image shifts of up to $10 \mu \mathrm{m}$. We have also shown that the amplitude of beam-image shift-induced coma depends elliptically on the direction of the shift and increases linearly with the amplitude of the shift, with slopes along the principal elliptical axes of 0.19 and $0.31 \mathrm{mrad}_{\mu} \mathrm{m}^{-1}$ (Supplementary Fig. S2c). These slopes are comparable to the values reported for Titan Krios microscopes (Thermo Fisher Scientific), although anisotropy of coma was not reported for Titan Krios (Cheng et al., 2018; Wu et al., 2019). The reasons for the azimuthal anisotropy of coma remain unknown and require further investigation. Here, we can only speculate that it may be caused by intrinsic properties of some of the optical elements or by inaccuracies in the adjustment of pivot points for image shift that result in beam tilt at the entrance to the incolumn $\Omega$ energy filter. Compensation for aberrations, using a simple function that depends linearly on image shifts, is efficient in correcting coma and to a lesser degree astigmatism (Figs. $2 b$ and $2 d$ ). The residual coma reveals a quasi-symmetric pattern, with its center of symmetry shifted off the coma-free center by a few micrometres.

The origin of such residual asymmetries is again not known. We believe that they may be caused by slight physical misalignment of condenser lenses or the in-column $\Omega$ energy filter.

Single-particle reconstruction of apoferritin with image shifts of up to $7 \mu \mathrm{m}$ shows that coma compensation is efficient and accurate to within $0.1 \mathrm{mrad}$ and that the residual astigmatism amplitude remains below $0.09 \mu \mathrm{m}$. No significant variation in higher-order aberrations associated with image shift can be detected at a resolution of $2 \AA$ (Supplementary Table S3). Collectively, this suggests that coma-corrected single-particle data collection delivers data with very similar quality and optical properties over a range of beam-image shifts with amplitudes of at least $7 \mu \mathrm{m}$. This also suggests that quick data collection does not compromise the quality of the single-particle data, at least to a resolution of $1.7 \AA$.

The benchmark data set was collected with a single image acquired per hole. In actual practice, however, three images are recorded per $1.2 \mu \mathrm{m}$ hole and five images are recorded per $2 \mu \mathrm{m}$ hole. For the most commonly used Quantifoil grids with hole patterns of R 0.6/1, R 1.2/1.3 and R 2/1, the number of images recorded (per position with an amplitude of $7 \mu \mathrm{m}$ ) varies between 30 and 125 . Thus, high-throughput collection can be achieved with all types of commonly used holey EM grids. It should be noted, however, that the choice of the pattern and the amplitude of the applied image shifts depend primarily on the availability of larger arrays of holes with homogeneous and optimal ice thickness.

With even larger image shifts of between 20 and $45 \mu \mathrm{m}$ it becomes possible to collect images for a complete grid square from a single stage position for 400- and 200-mesh grids. In this mode, hundreds of images can be recorded from a single stage position, potentially increasing throughput by an additional $20-50 \%$. Such an approach, however, requires more accurate coma and astigmatism correction, regular focusing and, even more importantly, the implementation of algorithms for the automated selection of optimal imaging areas. Consequently, procedures for applying beam-image shifts to variable patterns of holes for each selected square are also required.

An overnight data collection with a throughput exceeding 6000 images per day results in over 3000 images. For many cryo-EM samples, this represents a sufficient quantity of micrographs to produce a high-resolution reconstruction. At this data-collection rate, throughput is limited by the time needed to evaluate the quality of the cryo-EM grid, the collection of the grid atlas, the acquisition of square montages in SerialEM and the selection of holes with optimal ice thickness. These steps have already been automated in the EPU and Leginon software (Cheng et al., 2021). Implementing similar automation within the framework of SerialEM would further significantly boost microscope throughput.

While collecting benchmark data, we did not aim to achieve the highest resolution, but rather to characterize microscope behavior for data collected with large image shifts. Reconstruction of the collected data set reached a resolution of $1.7 \AA$, which corresponded to a 0.87 fraction of the Nyquist resolution. The resolution here was improved compared with our previous benchmark because of the larger number of particles, as well as denser particle packing in the holes, enabling more accurate refinement of CTF parameters and particle polishing. An improvement in resolution was achieved despite the use of carbon, rather than UltrAuFoil, grids and the use of a larger pixel size $(0.753 \AA$ versus $0.603 \AA)$. The Rosenthal-Henderson $B$ factor of $68 \AA^{2}$, which was calculated for the benchmark, is also comparable to the previously reported benchmark of $62 \AA^{2}$ (Fislage et al., 2020). It is also higher than the $B$ factor of $47 \AA^{2}$ reported for an apoferritin data set reconstituted to a resolution of $1.54 \AA$ from data recorded on a similar microscope, albeit at higher magnification (Kato et al., 2019). Multiple combinations of factors could account for the higher $B$ factor, including the type of grid used (carbon instead of UltrAuFoil), which resulted in higher initial drift caused by local charging (Russo \& Passmore, 2014). Suboptimal ice thickness could also be another factor out of many. As the resolution of the data set is close to the Nyquist limit, increasing the magnification may have further improved the resolution. Such improvement, however, would most likely have been moderate, because our data were collected in CDS mode on a K3 detector under near-optimal flux conditions, which have a high detective quantum efiiciency (DQE) up to the Nyquist resolution (Sun et al., 2020).

Interestingly, similar to the previous benchmark, the distribution of points on the $B$-factor plot is clearly curved (Fig. $3 d$ ), such that the $B$ factor decreases with increased resolution. This makes it more difficult to increase the resolution beyond the current limit. The reasons for this may include limited stability of the beam tilt (with approximately $\pm 0.1 \mathrm{mrad}$ observed on our microscope), as discussed previously (Fislage et al., 2020), and high average beam tilt. The latter may result in asymmetric aberrations that cannot be efficiently corrected beyond an achieved resolution. Combining a cFEG with an electron energy filter enables reconstruction at atomic resolution (Nakane et al., 2020; Yip et $a l ., 2020)$. The factors causing the bending of the $B$-factor plot 
will need to be understood and eliminated in order to be able to take advantage of the full potential of the CRYO ARM 300 for high-resolution imaging.

To conclude, we have shown that the CRYO ARM 300 is suitable for high-throughput data collection using multiple exposures per stage position by applying coma-andastigmatism-corrected beam-image shifts. The corrections are stable throughout the period of data collection. They are also accurate enough (for single-particle images acquired with beam-image shifts of up to at least $7 \mu \mathrm{m}$ ) to enable the 3D reconstruction of apoferritin to a resolution of $1.7 \AA$. The quality of single-particle data collected with such image shifts is indistinguishable from the quality of data collected on the coma-free axis. This enables a throughput exceeding 6000 images per day, and following the elimination of delays associated with setting beam-image shift, tilt and astigmatism, a throughput exceeding 10000 images per day was achieved for routine, high-resolution data collection on this microscope.

The final 3D map and raw data were deposited in the EMDB and EMPIAR databases with access codes EMD12358 and EMPIAR-10639.

\section{Acknowledgements}

We are grateful to Dr Adam Schröfel and Dr Marcus Fislage for technical support. We thank Dr Sohei Motoki (JEOL) for providing help with establishing procedures for the accurate alignment of pivot points and Dr David Mastronarde (University of Colorado, Boulder, Colorado, USA) for continuous support with SerialEM. The data were collected on a CRYO ARM 300 at the BECM facility of the VIB Center for Structural Biology, Brussels, Belgium.

\section{Funding information}

The following funding is acknowledged: Fonds Wetenschappelijk Onderzoek (grant No. G0H5916N to Rouslan Efremov); Vlaams Instituut voor Biotechnologie (award to Rouslan Efremov).

\section{References}

Afonine, P. V., Headd, J. J. \& Terwilliger, T. C. (2013). Comput. Crystallogr. Newsl. 4, 43-44.

Afonine, P. V., Klaholz, B. P., Moriarty, N. W., Poon, B. K., Sobolev, O. V., Terwilliger, T. C., Adams, P. D. \& Urzhumtsev, A. (2018). Acta Cryst. D74, 814-840.

Armstrong, M., Han, B.-G., Gomez, S., Turner, J., Fletcher, D. A. \& Glaeser, R. M. (2019). Biophys. J. 118, 708-719.
Cheng, A., Eng, E. T., Alink, L., Rice, W. J., Jordan, K. D., Kim, L. Y., Potter, C. S. \& Carragher, B. (2018). J. Struct. Biol. 204, 270-275.

Cheng, A., Negro, C., Bruhn, J. F., Rice, W. J., Dallakyan, S., Eng, E. T., Waterman, D. G., Potter, C. S. \& Carragher, B. (2021). Protein Sci. 30, 136-150.

Fislage, M., Shkumatov, A. V., Stroobants, A. \& Efremov, R. G. (2020). IUCrJ, 7, 707-718.

Glaeser, R. M., Typke, D., Tiemeijer, P. C., Pulokas, J. \& Cheng, A. (2011). J. Struct. Biol. 174, 1-10.

Grant, T., Rohou, A. \& Grigorieff, N. (2018). eLife, 7, e35383.

Hamaguchi, T., Maki-Yonekura, S., Naitow, H., Matsuura, Y., Ishikawa, T. \& Yonekura, K. (2019). J. Struct. Biol. 207, 40-48.

Kato, T., Makino, F., Nakane, T., Terahara, N., Kaneko, T., Shimizu, Y., Motoki, S., Ishikawa, I., Yonekura, K. \& Namba, K. (2019). Microsc. Microanal. 25, 998-999.

Mastronarde, D. N. (2005). J. Struct. Biol. 152, 36-51.

Mindell, J. A. \& Grigorieff, N. (2003). J. Struct. Biol. 142, 334-347.

Nakane, T., Kotecha, A., Sente, A., McMullan, G., Masiulis, S., Brown, P. M. G. E., Grigoras, I. T., Malinauskaite, L., Malinauskas, T., Miehling, J., Uchański, T., Yu, L., Karia, D., Pechnikova, E. V., de Jong, E., Keizer, J., Bischoff, M., McCormack, J., Tiemeijer, P., Hardwick, S. W., Chirgadze, D. Y., Murshudov, G., Aricescu, A. R. \& Scheres, S. H. W. (2020). Nature, 587, 152-156.

Penczek, P. A., Fang, J., Li, X., Cheng, Y., Loerke, J. \& Spahn, C. M. T. (2014). Ultramicroscopy, 140, 9-19.

Punjani, A., Rubinstein, J. L., Fleet, D. J. \& Brubaker, M. A. (2017). Nat. Methods, 14, 290-296.

Rohou, A. \& Grigorieff, N. (2015). J. Struct. Biol. 192, 216-221.

Rosenthal, P. B. \& Henderson, R. (2003). J. Mol. Biol. 333, 721-745.

Ruskin, R. S., Yu, Z. \& Grigorieff, N. (2013). J. Struct. Biol. 184, 385 393.

Russo, C. J. \& Passmore, L. A. (2014). Science, 346, 1377-1380.

Scheres, S. H. W. (2012). J. Struct. Biol. 180, 519-530.

Suloway, C., Pulokas, J., Fellmann, D., Cheng, A., Guerra, F., Quispe, J., Stagg, S., Potter, C. S. \& Carragher, B. (2005). J. Struct. Biol. 151, 41-60.

Sun, M., Azumaya, C., Tse, E., Southworth, D., Verba, K., Frost, A., Cheng, Y. \& Agard, D. (2020). Microsc. Microanal. 26, 1320.

Svensson, O., Malbet-Monaco, S., Popov, A., Nurizzo, D. \& Bowler, M. W. (2015). Acta Cryst. D71, 1757-1767.

Wagner, T., Merino, F., Stabrin, M., Moriya, T., Antoni, C., Apelbaum, A., Hagel, P., Sitsel, O., Raisch, T., Prumbaum, D., Quentin, D., Roderer, D., Tacke, S., Siebolds, B., Schubert, E., Shaikh, T. R., Lill, P., Gatsogiannis, C. \& Raunser, S. (2019). Commun. Biol. 2, 218.

Wu, C., Huang, X., Cheng, J., Zhu, D. \& Zhang, X. (2019). J. Struct. Biol. 208, 107396.

Wu, M., Lander, G. C. \& Herzik, M. A. Jr (2020). J. Struct. Biol. X, 4, 100020.

Yip, K. M., Fischer, N., Paknia, E., Chari, A. \& Stark, H. (2020). Nature, 587, 157-161.

Zheng, S. Q., Palovcak, E., Armache, J.-P., Verba, K. A., Cheng, Y. \& Agard, D. A. (2017). Nat. Methods, 14, 331-332.

Zivanov, J., Nakane, T., Forsberg, B. O., Kimanius, D., Hagen, W. J. H., Lindahl, E. \& Scheres, S. H. W. (2018). eLife, 7, e42166.

Zivanov, J., Nakane, T. \& Scheres, S. H. W. (2020). IUCrJ, 7, 253-267. 